\title{
INEQUALITIES FOR ORDER AND DISORDER PARAMETERS IN SU(N) GAUGE THEORIES
}

\author{
C.P. KORTHALS ALTES ${ }^{1}$ \\ Institute for Theoretical Physics, Princetonplein 5, P.O. Box 80006, 3508 TA Utrecht, The Netherlands \\ Received 30 July 1979 \\ (Final version received 3 December 1979)
}

\begin{abstract}
We discuss inequalities of the Mack-Fröhlich type in $\mathrm{SU}(N)$ gauge theories and their possible implications for the various phases of those theories.
\end{abstract}

\section{Introduction}

The long-distance behaviour of a pure $\mathrm{SU}(N)$ gauge theory (QCD) assigns a privileged rôle to its center group $\mathrm{Z}(N)$. The center group is the set of phases $\exp (i 2 \pi n / N)(n=0,1, \ldots, N-1)$.

t' Hooft [1] emphasized the importance of the center group in defining the electric (magnetic) flux tubes that eventually confine quarks (monopoles). His considerations led him in a natural way to introduce a disorder parameter, which is dual to the Wegner-Wilson $[2,3]^{\star}$ order parameter, when we restrict ourselves to the center group.

The behaviour of the (dis)order parameter characterizes the different phases of QCD. These phases can be realized by adding ad hoc scalar fields to the lagrangian of QCD, that break the gauge symmetry completely (magnetic confinement phase), partially (phases with long-range forces) or not at all (electric confinement phase). Instead of adding these symmetry-breaking fields explicitly one can think of them as being dynamically generated by the gluon fields; they should be built up in terms of the gauge-field strength tensor $G_{\mu \nu}^{a}$ and are therefore insensitive to center-group transformations.

One can experiment with these ideas on a lattice à la Wilson [2]. Admitting only symmetry-breaking fields that do not transform under the center group $Z(N)$, it is possible to freeze all the group degrees of freedom, except the $N$ degrees of freedom of $Z(N)$. This limit is the one where all gluons have acquired an infinite mass. To be

${ }^{1}$ Permanent address: Centre Physique Théorique du CNRS, Route Léon Duchamps, Campus de Luminy, Marseille, France.

* For later work on plaquette models, see [3a-d]. 
specific: in the case of SU(2) we add two isotriplet scalar fields with only angular degrees of freedom and with vacuum expectation values $a$ and $b$. Letting $a$ go to infinity freezes the $S U(2)$ gauge field down to a $U(1)$ gauge field. Letting in addition, $b$ to go infinity freezes $U(1)$ down to $Z(2) . Z(N)$ gauge theory has been studied by several authors [3]. The important property of these "mini gauge theories" is that there is an exact symmetry called duality that is of great help in elucidating their phase structure. It turns out that for a special one-parameter family of $Z(N)$ gauge theories the duality transformation is particularly simple [3], with one fixed point.

For low values of $N$ there are two phases: an electric confinement phase below the fixed point with a condensate of magnetic vertices as ground state, and above the fixed point a magnetic confinement phase with a condensate of electric vertices as ground-state. For high values of $N$ there is a region around the fixed point, where condensation of electric and magnetic vertices is inhibited. There we have a third phase with a long-range force $[12]^{\star}$.

An interesting idea, quite natural in this context, is due to Mack and Petkova [4]. The idea is to relate the (dis)order parameter of the full $S U(N)$ group to that of its center group. They realized this idea in the case of a restricted SU(2) lattice gauge theory. Fröhlich [5] realized that one could dispense with the restriction and related the Wilson order parameter for any gauge theory to that of its center group. Here we also want to write down relations for the disorder parameter and to sharpen the results obtained in [5]. The main points are:

(i) if electric confinement in $\mathrm{Z}(N)$ is realized up to $K=K_{\mathrm{c}}$, then electric confinement in $\mathrm{SU}(N)$ is realized up to $K_{\mathrm{c}} / N$;

(ii) if electric confinement is $\mathrm{SU}(2)$ is realized up to $\frac{1}{2} K_{\mathrm{c}}$, then magnetic confinement is excluded up to $\frac{1}{2} K_{\mathrm{c}}$.

These two statements are based upon the inequalities proven in sects. 2,3 , respectively:

$$
\langle A(\mathrm{C})\rangle_{\mathrm{SU}(N)}(K) \leqslant\langle A(\mathrm{C})\rangle_{\mathrm{Z}(N)}(N K)
$$

for the order parameters $A(C)$, and

$$
\langle B(\mathrm{C})\rangle_{\mathrm{SU}(2)}(K) \geqslant\langle B(\mathrm{C})\rangle_{\mathrm{Z}(2)}(2 K),
$$

for the disorder parameter $B(C)$. The notation $\langle Y\rangle_{\mathrm{X}}$ means computing the average of quantity $Y$ within the gauge group $\mathrm{X}$.

\section{Inequality for the order parameter}

In this section we want to give a very simple explanation why

$$
\langle A(C)\rangle_{\mathrm{SU}(N)}(K) \leqslant\langle A(C)\rangle_{\mathrm{Z}(N)}(N K) .
$$

\footnotetext{
* See the fourth of these papers for a computer calculation.
} 
The left-hand side of eq. (2.1) is the Wegner [3]-Wilson [2] order parameter and is evaluated on a hypercubic lattice $\mathrm{L}$ in an arbitrary number of dimensions:

$$
\langle A(\mathrm{C})\rangle_{\mathrm{SU}(N)}(K) \equiv \frac{1}{Q} \int \prod_{\ell \in \mathrm{L}} \mathrm{d} U(\ell) \frac{1}{N} \operatorname{Tr} U(\mathrm{C}) \exp S(K ; U)
$$

$S(K, U)$ is the well-known Wilson lattice action [2] for $\mathrm{SU}(N)$ :

$$
S=\frac{1}{2} K \sum_{\mathrm{P}}[\operatorname{Tr} U(\mathrm{P})+\text { c.c. }] \text {. }
$$

Here $\mathrm{P}$ is a two-dimensional plaquette in $\mathrm{L}, \sum_{\mathrm{P}}$ means summation over all such plaquettes, and $U(\mathrm{P}) \equiv \prod_{\ell-\mathrm{P}} U(\ell)$. $\ell$ stands for any link, $U(\ell)$ is an $\operatorname{SU}(N) N \times N$ matrix, and $\mathrm{C}$ is a closed simple curve on $\mathrm{L} . U(\mathrm{C})$ stands for $\prod_{\ell \rightarrow \mathrm{C}} U(\ell)$ and all products of $\mathrm{U}(\ell)$ are ordered. The coupling constant $K$ is supposed to be nonnegative ("ferromagnetic"). The partition function $Q=Q(K)$ is the familiar

$$
Q(K)=\int \prod_{\ell \leftarrow \mathbf{L}} \mathrm{d} U(\ell) \exp S(K ; U)
$$

$\mathrm{By}\langle A(\mathrm{C})\rangle_{\mathrm{Z}(N)}(K)$ we mean the Wegner [3] parameter

$$
\langle A(\mathrm{C})\rangle_{\mathrm{Z}(N)}(K)=\frac{1}{Q_{\mathrm{Z}(N)}} \sum_{\{z(\ell)\}} z(\mathrm{C}) \exp S(K ; z),
$$

with $S(K ; z)$ the $Z(N)$ action:

$$
S(K ; z)=\frac{1}{2} K\left[\sum_{\mathrm{P}} z(\mathrm{P})+\text { c.c. }\right] .
$$

Here we denote by $z(\mathrm{P})=\prod_{\ell \leftarrow \mathrm{P}} z(\ell), z(\ell)$ is a complex number with $[z(\ell)]^{N}=1$, $z(\mathrm{C}) \equiv \prod_{\ell \leftarrow \mathrm{C}} z(\ell)$ and $Q_{\mathrm{Z}(N)}$ is the corresponding partition function. This fixes our notation.

Then we can easily relate the two order parameters (2.2) and (2.5) by considering the $\mathrm{SU}(N)$ action $S(K ; U)$ as a $\mathrm{Z}(N)$ action with fluctuating couplings: $S(K: U) \equiv$ $S\left(K_{\mathrm{P}} ; z\right)$, with of course $K_{\mathrm{P}}=K \operatorname{Tr} U(\mathrm{P})$; to this end consider a fixed configuration of $\mathrm{Z}(N)$ elements on the links of $\mathrm{L}:\{z(\ell)\}$. Define a new $\mathrm{SU}(N)$ matrix $\hat{U}(\ell)$ :

$$
U(\ell)=\hat{U}(\ell) z(\ell) \text {. }
$$

It follows that

$$
\langle A(\mathrm{C})\rangle_{\mathrm{SU}(N)}(K)=\frac{\int \prod_{\ell \leftarrow \mathrm{L}} \mathrm{d} \hat{U}(\ell)(1 / N) \operatorname{Tr} \hat{U}(\mathrm{C}) z(\mathrm{C}) \exp S\left(\hat{K}_{\mathrm{P}}: Z\right)}{\int \prod_{\ell-\mathrm{L}} \mathrm{d} \hat{U}(\ell) \exp S\left(\hat{K}_{\mathrm{P}} ; z\right)},
$$

where we cancelled a common factor $\prod_{\ell \leftarrow L} z(\ell)$ in numerator and denominator. Since the left-hand side does not depend on $\{z(\ell)\}$ we can sum numerator and 
denominator over all possible $\mathrm{Z}(N)$ configurations $\{z(\ell)\}$ and obtain

$$
\langle A(\mathrm{C})\rangle_{\mathrm{SU}(N)}(K)=\frac{\int \prod_{\ell-\mathrm{L}} \mathrm{d} \hat{U}(\ell)(1 / N) \operatorname{Tr} \hat{U}(\mathrm{C})\langle A(\mathrm{C})\rangle_{\mathbf{Z}(N)}\left(\hat{K}_{\mathrm{P}}\right) Q_{\mathrm{Z}(N)}\left(\hat{K}_{\mathrm{P}}\right)}{\int \prod_{\ell+\mathrm{L}} \mathrm{d} \hat{U}(\ell) Q_{\mathbf{Z}(N)}\left(\hat{K}_{\mathrm{P}}\right)},
$$

with $\hat{K}_{\mathrm{P}} \equiv K \operatorname{Tr} \hat{U}(\mathrm{P})$, using definition (2.5).

This relation is useful if we are able to give a bound for $\langle A(C)\rangle_{Z(N)}\left(\hat{K}_{\mathrm{P}}\right)$ which is uniform in $\hat{K}_{\mathrm{P}}$. By using an elegant refinement [8] of inequalities for ferromagnetic systems $[6,7]$, this is indeed possible. We should mention that ref. [5] follows approximately the same method but our result is slightly improved. The point is now the following: the $\hat{K}_{\mathrm{P}}$ are no longer ferromagnetic: in fact for $N \geqslant 3$ they can be complex. Now the old inequalities [6] said that two ferromagnetic systems (with couplings $0 \leqslant K_{\mathrm{P}} \leqslant K_{\mathrm{P}}^{\prime}$ ) had correlation functions $C_{K_{\mathrm{P}}}$ and $C_{K_{\mathrm{P}}^{\prime}}$ obeying

$$
C_{K_{\mathrm{P}}} \leqslant C_{K_{\mathrm{P}}^{\prime}},
$$

which is physically obvious: strengthening ferromagnetic couplings tend to strengthen correlations.

Ginibre [7] realized that the system with $K_{\mathrm{P}}$ couplings could be antiferromagnetic, eq. (2.9a) still holding provided

$$
\left|K_{\mathrm{P}}\right| \leqslant K_{\mathrm{P}}^{\prime}
$$

This case applies to $N=2$ but for $N \geqslant 3$ we need the refinement of ref. [8]. Let us write the action as

$$
S\left(\hat{K}_{\mathrm{P}}, z\right)=\sum_{\mathrm{P}}\left|\hat{K}_{\mathrm{P}}\right| \cos (\zeta(\mathrm{P})+\alpha(\mathrm{P})),
$$

where $\zeta(\mathrm{P})$ is the argument of $z(\mathrm{P})$ and $\alpha(\mathrm{P})$ the argument of $K_{\mathrm{P}}$. Then according to ref. [8]

$$
\langle\cos \zeta(\mathrm{C})+\alpha(\mathrm{C}))\rangle_{\mathbf{Z}(N)}\left(\left|\hat{K}_{\mathrm{P}}\right|, \alpha(\mathrm{P})\right) \leqslant\langle\cos \zeta(\mathrm{C})\rangle,\left(\left|\hat{K}_{\mathrm{P}}\right|\right)
$$

We can use it in eq. (2.8) by writing the two factors in the middle of the numerator of (2.8) differently:

$$
\frac{1}{N} \operatorname{Tr} \hat{U}(\mathrm{C})\langle A(\mathrm{C})\rangle_{\mathrm{Z}(N)}\left(\hat{K}_{\mathrm{P}}\right)=\frac{1}{N}|\operatorname{Tr} \hat{U}(\mathrm{C})|\left\langle\cos (\zeta(\mathrm{C})+\alpha(\mathrm{C})\rangle\left(\left|K_{\mathrm{P}}\right|, \alpha(\mathrm{P})\right) .\right.
$$

Therefore ( $2.9 \mathrm{~b})$ says

$$
\frac{1}{N} \operatorname{Tr} \hat{U}(\mathrm{C})\langle A(\mathrm{C})\rangle_{\mathbf{Z}(N)}\left(\hat{K}_{\mathrm{P}}\right) \leqslant \frac{1}{N}|\operatorname{Tr} \hat{U}(\mathrm{C})|\langle\cos (\zeta(\mathrm{C}))\rangle\left(\left|\hat{K}_{\mathrm{P}}\right|\right),
$$

and using the old inequalities $(2.9 \mathrm{a})$ :

$$
\langle\cos \zeta(\mathrm{C})\rangle_{\mathrm{Z}(N)}\left(\left|\hat{K}_{\mathrm{P}}\right|\right) \geqslant\langle\cos \zeta(\mathrm{C})\rangle_{\mathrm{Z}(N)}(N K),
$$


since $\left|\hat{K}_{\mathrm{P}}\right| \leqslant N K$. Obviously $(1 / N)|\operatorname{Tr} \hat{U}(\mathrm{C})| \leqslant 1$ and therefore, combining (2.10a), $(2.10 \mathrm{~b})$, and substituting into (2.8) we obtain (2.1), as promised. The amount of physics, special to gauge fields, going into this result is little; it may therefore well be that the bound (especially the factor $N$ in front of $K$ in the r.h.s. of (2.1)) can be ameliorated considerably. (Note that we have a factor $N$ instead of $2 N$ as in ref. [5].)

\section{Bound for the disorder parameter}

In this section we will consider the disorder parameter first introduced by ' $t$ Hooft [1] and its relation to the disorder parameter in $\mathrm{Z}(N)$ gauge theory. Let us first state the result:

$$
\left\langle B\left(C^{\mathrm{d}}\right)\right\rangle_{\mathrm{SU}(2)}(K) \geqslant\left\langle B\left(\mathrm{C}^{\mathrm{d}}\right)\right\rangle_{\mathrm{Z}(2)}(2 K) .
$$

It is the same result as for the restricted $\mathrm{SU}(2)$ theory in ref. [4].

First recall the definition of $\left\langle B\left(C^{d}\right)\right\rangle$ working for the sake of definiteness in four dimensions: let $C^{d}$ be a closed simple curve on the dual lattices $L^{d}$; consider a surface $\Sigma\left(C^{\mathrm{d}}\right)$ on $L^{\mathrm{d}}$ subtended by $\mathrm{C}^{\mathrm{d}}$. Consider the set of plaquettes on $L$ dual to $\left(\mathrm{C}^{\mathrm{d}}\right)$ and call it $\left[\Sigma\left(C^{d}\right)\right]^{*}$. Then

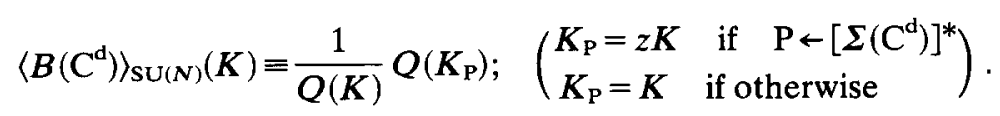

Here $z$ is a fixed element of $Z(N), \Sigma\left(C^{d}\right)$ can be varied by redefining link variables, so $\left\langle B\left(C^{d}\right)\right\rangle$ only depends on $C^{d}$.

After having gone through the same manipulations as those leading to eq. (2.8) we obtain

$$
\langle B(\mathrm{C})\rangle_{\mathrm{SU}(N)}(K)=\frac{1}{\hat{Q}(K)} \int \prod_{\ell \in \mathrm{L}} \mathrm{d} \hat{U}(\ell)\left\langle B\left(\mathrm{C}^{\mathrm{d}}\right)\right\rangle_{\mathrm{Z}(N)}\left(\hat{K}_{\mathrm{P}}\right) Q_{\mathrm{Z}(N)}\left(\hat{K}_{\mathrm{P}}\right),
$$

with

$$
\hat{Q}(K) \equiv \int \prod_{\ell \in \mathrm{L}} \mathrm{d} \hat{U}(\ell) Q_{\mathbf{Z}(N)}\left(\hat{K}_{\mathrm{P}}\right) .
$$

The quantity $\left\langle B\left(C^{d}\right)\right\rangle$ is defined as in eq. (3.2), except that $Q\left(K_{\mathrm{P}}\right)$ is replaced by $Q_{\mathrm{Z}(N))}\left(K_{\mathrm{P}}\right)$. Of course we have again $\hat{K}_{\mathrm{P}} \equiv K \operatorname{Tr} \hat{U}(\mathrm{P})$.

Let us first consider the case $N=2$. Duality in the sense of the group $Z(2)$ permits us to relate order and disorder parameters [3]:

$$
\left\langle B\left(\mathrm{C}^{\mathrm{d}}\right)\right\rangle_{\mathbf{Z}(2)}\left(\hat{K}_{\mathrm{P}}\right)=\left\langle A\left(\mathrm{C}^{\mathrm{d}}\right)\right\rangle_{\mathbf{Z}(2)}\left(\hat{K}_{\mathrm{P}}^{*}\right),
$$

where

$$
\hat{K}_{\mathrm{P}}^{*}=-\frac{1}{2} \log \text { th } \hat{K}_{\mathrm{P}}=-\frac{1}{2} \log \text { th }\left|\hat{K}_{\mathrm{P}}\right|+\frac{1}{2} i \pi \frac{1}{2}\left(1-\operatorname{sign} \hat{K}_{\mathrm{P}}\right) .
$$

So we have to find a bound for $\left\langle A\left(C^{d}\right)\right\rangle_{Z(2)}$ when we have possibly complex coupling. 
In the case at hand $(\mathrm{SU}(2))$ this is not a series complication since $\operatorname{Tr} \hat{U}(\mathrm{P})$ has its phase in $Z(2)$. Therefore $\hat{K}_{\mathrm{P}}$ is just a dislocation [10], if sign $\operatorname{Tr} \hat{U}(\mathrm{P})<0$. Let the set of plaquettes where $\hat{K}_{\mathrm{P}}^{*}$ has the imaginary part $\frac{1}{2} i \pi$ be denoted by $\mathrm{T}$. Then

$$
\left\langle\mathrm{A}\left(\mathrm{C}^{\mathrm{d}}\right)\right\rangle_{\mathrm{Z}(2)}\left(\hat{K}_{\mathrm{P}}^{*}\right)=\frac{\sum_{\{z(\ell)\}} z\left(\mathrm{C}^{\mathrm{d}}\right) i^{|T|} \prod_{\mathrm{P} \leftarrow \mathrm{T}} z(\mathrm{P}) \exp S\left(\left|\hat{K}_{\mathrm{P}}\right|^{*}, z\right)}{\sum_{\{z(\ell)\}} i^{|T|} \prod_{\mathrm{P} \leftarrow \mathrm{T}} z(\mathrm{P}) \exp S\left(\left|\hat{K}_{\mathrm{P}}\right|^{*}, z\right)} .
$$

Cancel the powers $i^{|T|}(|T|$ is the number of plaquettes in $\mathrm{T})$ and divide both numerator and denominator by $Q_{\mathrm{Z}(2)}\left(\left|\hat{K}_{\mathrm{P}}\right|^{*}\right)$ :

$$
\left\langle A\left(\mathrm{C}^{\mathrm{d}}\right)\right\rangle_{\mathrm{Z}(2)}\left(\hat{K}_{\mathrm{O}}^{*}\right)=\frac{\left.\left\langle z\left(\mathrm{C}^{\mathrm{d}}\right) \prod_{\mathrm{P} \leftarrow \mathrm{T}} z(\mathrm{P})\right\rangle_{\mathrm{Z}(2)}\left|\hat{K}_{\mathrm{P}}\right|^{*}\right)}{\left\langle\prod_{\mathrm{P} \leftarrow \mathrm{T}} z(\mathrm{P})\right\rangle_{\mathrm{Z}(2)}\left(\left|\hat{K}_{\mathrm{P}}\right|^{*}\right)} .
$$

The last expression relates $\langle A(C)\rangle\left(\hat{K}_{\mathrm{P}}^{*}\right)$ to a correlation function and this is precisely due to $\hat{K}_{\mathrm{P}}$ being a dislocation on $\mathrm{T}$. But now we can use the original Griffiths inequalities [6] to obtain

$$
\begin{aligned}
\frac{\left\langle z\left(\mathrm{C}^{\mathrm{d}}\right) \prod_{\mathrm{P}-\mathrm{T}} z(\mathrm{P})\right\rangle_{\mathrm{Z}(2)}\left(\left|\hat{K}_{\mathrm{P}}\right|^{*}\right)}{\left\langle\prod_{\mathrm{P} \leftarrow \mathbf{T}} z(\mathrm{P})\right\rangle_{\mathbf{Z}(2)}\left(\left|\hat{K}_{\mathrm{P}}\right|^{*}\right)} & \geqslant\left\langle z\left(\mathrm{C}^{\mathrm{d}}\right)\right\rangle\left(\left|\hat{K}_{\mathrm{P}}\right|^{*}\right) \\
& =\langle A(\mathrm{C})\rangle\left(\left|\hat{K}_{\mathrm{P}}\right|^{*}\right),
\end{aligned}
$$

and since

$$
\left|\hat{K}_{\mathrm{P}}\right|^{*} \geqslant(2 K)^{*}
$$

we obtain

$$
\langle A(C)\rangle_{\mathbf{Z}(2)}\left(\hat{K}_{\mathrm{P}}^{*}\right) \geqslant\left\langle A\left(C^{\mathrm{d}}\right)\right\rangle_{\mathbf{Z}(2)}\left((2 K)^{*}\right)=\left\langle B\left(C^{\mathrm{d}}\right)\right\rangle_{\mathbf{Z}(2)}(2 K),
$$

and (3.1) follows if $N=2$.

The SU(3) case is treated up to and including eq. (3.4) in the same way. But now $\hat{K}_{\mathrm{P}}$ can have any phase; it is only true that $\left|\hat{K}_{\mathrm{P}}\right|$ takes its maximum value in the center $\mathrm{Z}(3)$. Eq. (3.7) becomes more complicated, namely a sum of correlation functions.

The prcise form of the analogue of eq. (3.6) for the case $N=3$ is

$$
\begin{aligned}
& \left\langle A\left(\mathrm{C}^{\mathrm{d}}\right)\right\rangle_{\mathrm{Z}(3)}\left(\hat{K}_{\mathrm{P}}^{*}\right) \\
& =\frac{\sum_{\{z(\ell)\}} z\left(\mathrm{C}^{\mathrm{d}}\right) \prod_{\mathrm{P} \leftarrow \mathrm{T}}\left(X_{0}\left(\phi_{\mathrm{P}}\right)+X_{1}\left(\phi_{\mathrm{P}}\right) z(\mathrm{P})+X_{2}\left(\phi_{\mathrm{P}}\right) \bar{z}(\mathrm{P})\right) \exp S\left(\left|\hat{K}_{\mathrm{P}}\right|^{*}, z\right)}{\sum_{\{z(\ell)\}} \Pi_{\mathrm{P} \leftarrow \mathrm{T}}\left(X_{0}\left(\phi_{\mathrm{P}}\right)+X_{1}\left(\phi_{\mathrm{P}}\right) z(\mathrm{P})+X_{2}\left(\phi_{\mathrm{P}}\right) \bar{z}(\mathrm{P})\right) \exp S\left(\left|\hat{K}_{\mathrm{P}}\right|^{*}, z\right)} .
\end{aligned}
$$

The functions $X_{i}\left(\phi_{\mathrm{P}}\right)$ also depend on $\left|\hat{K}_{\mathrm{P}}\right| X_{1}$ and $X_{2}$ are translates of $X_{0}$ :

$$
X_{1}\left(\phi_{\mathrm{P}}\right)=X_{0}\left(\phi_{\mathrm{P}}-\frac{2}{3} \pi\right), \quad X_{2}\left(\phi_{\mathrm{P}}\right)=X_{0}\left(\phi_{\mathrm{P}}-\frac{4}{3} \pi\right) .
$$

If $\phi_{\mathrm{P}}=\frac{2}{3} \pi$ or $\phi_{\mathrm{P}}=\frac{4}{3} \pi$ we have the usual result [3d] for dislocations in $\mathrm{Z}(3)$ :

$$
X_{0}\left(\frac{2}{3} \pi\right)=X_{0}\left(\frac{4}{3} \pi\right)=0, \quad X_{0}(0)=1,
$$

and $X_{0}$ is a symmetric function of $\phi_{\mathrm{P}}$. For a definition of the function $X_{0}(\phi)$ see beneath fig. 2 . In fig. 2 we have plotted $X_{0}(\phi)$ for some values of $|\hat{K}|$. The difficulty 
13

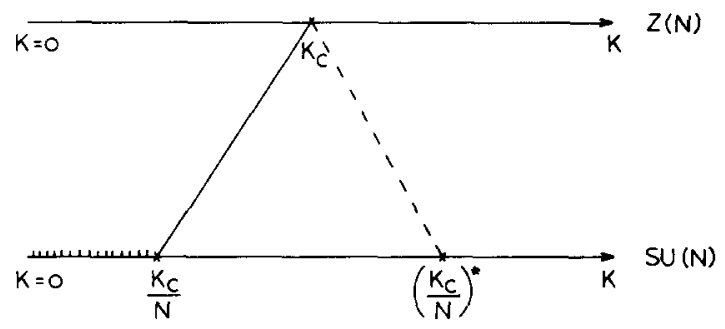

Fig. 1. The unbroken line represents the relation (1.1). The crosshatched region on the left is the region where confinement is certainly realized, if $Z(N)$ theory confines up to $K_{\mathrm{c}}$. The broken line represents the unproved relation (4.1). On the right of the point $\left(K_{c} / N\right)^{*}$ we would then definitely have magnetic confinement.

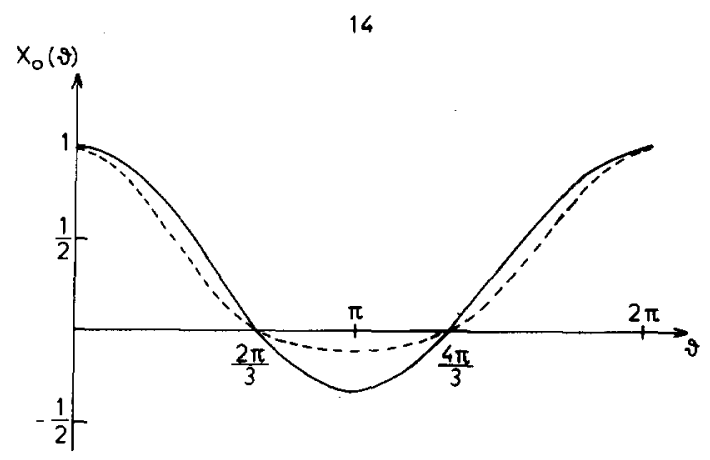

Fig. 2. The function $X_{0}(\phi)$ on the interval. Unbroken line: $X_{0}(\phi)$ if $|\hat{K}|=10^{-4}$; broken line: $X_{0}(\phi)$ if $|\hat{K}|=1 . X_{0}(\phi)$ is defined in terms of the functions $I_{\eta}(\hat{K})$ :

$$
\mathrm{e}^{\left(\hat{K}_{z}+\bar{K}_{\bar{z}}\right) / 2}=I_{0}(\hat{K})+I_{1}(\hat{K}) z+I_{2}(\hat{K}) \bar{z}
$$

where $\hat{K}=|\hat{K}| \mathrm{e}^{i \phi}$. Then $X_{0}(\phi)=\frac{1}{3} \sum_{\eta=0}^{2} I_{\eta}(K) / I_{\eta}(|\hat{K}|)$.

with eq. (3.6') is, that none of the inequalities in ref. [9] is applicable. The bound (1.2) for the SU(3) case therefore involves more dynamics, if it exists at all. On the basis of the results in ref. [11] we would tend to believe it to be true.

\section{Discussion}

The bounds or the order parameters (1.1) and (1.2) are very useful. They tell us where electric confinement is sure to occur for $\operatorname{SU}(N)$ once we know where it occurs for $\mathrm{Z}(N)$. From the inequality (1.2) we can exclude the possibility that magnetic confinement occurs, where electric confinment is guaranteed by (1.1). Let us briefly discuss the phases of $Z(N)$ [3]. For $N=2$ and $N=3$ there is just one parameter $K$ and duality tells us where the possible transition point $K_{c}$ is. Barring the existence of other transition points, the phase diagram for $Z(N)$ is very simple (see fig. 1 upper 
line). The electric confinement region for $K<K_{\mathrm{c}}$ is separated from the magnetic confinement region for $K>K_{\mathrm{c}}$. On the lower line the coupling constant $K$ is plotted for $\mathrm{SU}(N)$. For $K<K_{\mathrm{c}} / N$ we are sure of electric confinement. For $N=2$ we cannot have magnetic confinement for $K<\frac{1}{2} K_{\mathrm{c}}$ as follows from eq. (1.2).

For $N=3$ one expects from 't Hooft arguments [11] that, like in the $N=2$ case, electric confinement excludes magnetic confinement. So one might expect eq. (1.2) to be true for $N=3$.

What happens for large $N$ ? As $N \geqslant 4$ the coupling parameter space becomes multidimensional. Still there is a one-parameter family which can be written as a Villain-type theory [3d] and which is self-dual. The ensuing critical coupling $K_{\mathrm{c}}$ is proportional to $N$, so for large $N$ the region where (1.1) predicts confinement remains of finite extension.

Another question, more difficult to answer, is: can we find bounds like (1.1) for the disorder parameter $\langle B(C)\rangle_{\mathrm{SU}(N)}$ ? If so we would be able to predict where magnetic confinement is sure to occur. Let us speculate for a moment that there might be a duality argument for the full gauge group. Remember that in $\mathrm{Z}(N)$ the order parameter $\langle A(C)\rangle_{\mathbf{Z}(2)}(K)$ equals the disorder parameter evaluated at the dual coupling $K^{*}$. This is valid for any loop $\mathrm{C}$ no matter how large or small $\mathrm{C}$ is. It may be that only for very large contours $\mathrm{C}$ such a relation becomes true for $\mathrm{SU}(N)$. Then we would have a bound of the form

$$
\langle B(\mathrm{C})\rangle_{\mathrm{SU}(N)}(K) \leqslant\langle B(\mathrm{C})\rangle\left((N K)^{*}\right)
$$

which, again, is mere speculation. The result is depicted in fig. 1 by the dotted line. To the right of the point $\left(K_{\mathrm{c}} / N\right)^{*}$ we would have magnetic confinement. In between $K_{\mathrm{c}} / N$ and $\left(K_{\mathrm{c}} / N\right)^{*}$ there is still room for the "Georgi-Glashow" self-dual phase discussed in ref. [11]. The continuum theory for SU(2) would then be (in terms of $\left.\alpha \equiv g^{2} / 4 \pi\right)$ as follows.

(i) Higgs phase if $\alpha \leqslant \alpha_{1}$ :

$$
-\log A(\mathrm{C}) \sim|\mathrm{C}|, \quad-\log B(\mathrm{C}) \sim|\Sigma(\mathrm{C})| .
$$

(ii) Self-dual partial Higgs phase for $\alpha_{1} \leqslant \alpha \leqslant \alpha_{2}=\alpha_{1}^{-1}$ :

monopole charge $4 \pi / g$ and charge $g$ are dual so $\alpha=g^{2} / 4 \pi=1$ is a symmetry point within this phase:

$$
-\log A(\mathrm{C}) \sim-\log B(\mathrm{C}) \sim|C| .
$$

This phase may be absent, i.e. $\alpha_{1}=\alpha_{2}=1$.

(iii) Confinement phase if $\alpha \geqslant \alpha_{2}$ :

$$
-\log A(\mathrm{C}) \sim|\Sigma(\mathrm{C})|, \quad-\log B(\mathrm{C}) \sim|\mathrm{C}| .
$$

The transition points should be such that the $\beta$-function has no zeros (so the transition should not be second order). All of this is just amusing speculation. 
A final word about the inequalities (1.1) and (1.2). If a continuum limit for $Z(N)$ and $\mathrm{SU}(N)$ gauge theory exists, then they should hold in that limit. However, for low $N$, the results of ref. [9] suggest that the transition is first order for $\mathrm{Z}(N)$. It is not known how to construct the continuum theory in such a case.

I am indebted to Prof. G. 't Hooft, Dr. J. Groeneveld, Dr. J. Jurkiewicz and Dr. A. Messager for discussions.

\section{References}

[1] G. 't Hooft, Nucl. Phys. B138 (1978) 1.

[2] K. Wilson, Phys. Rev. D10 (1974) 2445.

[3] F. Wegner, J. Math. Phys. 12 (1971) 2259.

[3a] R. Balian, J. M. Drouffe and C. Itzykson, Phys. Rev. D11 (1978) 2098.

[3b] T. Voneya, preprint NCC.

[3c] C.P. Korthals Altes, Nucl. Phys. B142 (1978) 315.

[3d] A. Casher, Tel Aviv preprint (1978).

[4] G. Mach and V. B. Petkova, Hamburg preprint (Dec., 1978).

[5] J. Fröhlich, I.H.E.S. preprint (Feb., 1978).

[6] R.B. Griffiths, J. Math. Phys. 8 (1967) 478.

[7] J. Ginibre, Comm. Math. Phys. 16 (1970) 310.

[8] A. Messager, S. Miracle-Sole and C. Pfister, Comm. Math. Phys. 58 (1978) 19.

[9] M. Creutz, L. Jacobs and C. Rebbi, Brookhaven preprint (April, 1979).

[10] L.P. Kadanoff and H. Ceva, Phys. Rev. B3 (1971) 3918.

[11] G. 't Hooft, Utrecht preprint (1979).

[12] S. Elitzur, R.B. Pearson and J. Shigemitsu, IAS preprint (1979);

D. Horn, M. Weinstein and S. Jankielowicz, preprint (1979);

A. Ukawa, P. Windey and A.H. Guth, IAS preprint (1979);

M. Creutz, L. Jacobs and C. Rebbi, Brookhaven preprint (June, 1979). 